\title{
Effects of Neuromuscular Electrical Stimulation on Arterial Hemodynamic Properties and Body Composition in Paretic Upper Extremities of Patients with Subacute Stroke
}

\author{
Shu-Chun Huang ${ }^{1}$, Alice May-Kuen Wong ${ }^{1}$, Yu-Fen Chuang ${ }^{2}$, Yu-Chuan Liu ${ }^{1}$, Wei-Lun Tsai ${ }^{1}$, \\ Jong-Shyan Wang ${ }^{2}$
}

Background: Neuromuscular electric stimulation (NMES) induces repeated muscular contraction, possibly promoting the perfusion/oxygenation of the regional tissues. It remains unclear how NMES influences vascular hemodynamic property and segmental fluid distribution/composition in paretic extremities of hemiplegic patients.

Methods: $\quad$ Eleven hemiplegic patients aged $62.6 \pm 12.5$ years in the subacute stage of stroke received NMES for paretic wrist extensor and flexor muscles 30 min daily, 5 days per week for 4 weeks. The non-paretic upper extremities (NPUE) that did not receive NMES served as control. Distribution of fluid to intra/extracellular milieu and arterial hemodynamic properties were determined by using the multi-frequency bioelectrical impedance and pulse wave analysis, respectively.

Results: $\quad$ Compared with NPUE without NMES, paretic upper extremity (PUE) with NMES revealed a significantly less decrease in arterial blood flow, impedance quotient, slope quotient, and less increase in crest width and crest time of arterial pulse wave. NMES for 4 weeks increased body cell mass in PUE. Furthermore, NPUE without NMES reduced intracellular water, whereas PUE with NMES retarded loss of intracellular water after stroke.

Conclusion: NMES therapy increases body cell mass, attenuates reduc-

\section{At a Glance Commentary}

Scientific background of the subject

Neuromuscular electric stimulation (NMES) induces repeated passive muscular contraction, promoting the perfusion/oxygenation of the regional tissues. Investigation regarding the effect of a repeated NMES intervention protocol on segmental fluid distribution, chemical composition and hemodynamic properties of hemiplegic extremities following stroke are still lacking.

\section{What this study adds to the field}

Repeated NMES treatment delays stroke-related decline of peripheral vascular function and segmental fluid redistribution in the paretic upper extremities of patients with hemiplegia. It also maintains the regional tissue in a favorable nutritional status. Additionally, resistance training for the non-paretic extremities may be included in the rehabilitation program to attenuate the decline of hemodynamic function. tion of intracellular water, and alleviates arterial hemodynamic disturbance in PUE in subacute stroke. However, stroke-related physical deconditioning may negatively regulate body composition and impair hemodynamic function in NPUE.

(Biomed J 2014;37:205-210)

Key words: body composition, cellular fluid, hemodynamics, neuromuscular electrical stimulation, stroke

\footnotetext{
From the ${ }^{1}$ Department of Physical Medicine and Rehabilitation, Chang Gung Memorial Hospital at Linkou, Chang Gung University College of Medicine, Taoyuan, Taiwan; ${ }^{2}$ Graduate Institute of Rehabilitation Science, College of Medicine, Chang Gung University, Taoyuan, Taiwan

Received: Mar. 03, 2013; Accepted: Aug. 05, 2013

Correspondence to: Prof. Jong-Shyan Wang, Graduate Institute of Rehabilitation Science, College of Medicine, Chang Gung University. 259 Wenhua $1^{\text {st }}$ Rd., Gueishan, Taoyuan, 333, Taiwan (R.O.C.). Tel: 886-3-2118800 ext. 5748; Fax: 886-3-2118700;

E-mail: s5492@mail.cgu.edu.tw
}

DOI: $10.4103 / 2319-4170.117892$ 
L imb deconditioning impairs endothelial vascular control, decreases vessel compliance, and subsequently causes vascular atrophy. ${ }^{[1,2]}$ Although hemiplegic extremities after stroke may depress the vascular tone by autonomic vasomotor dysfunction, prolonged inactivity in the paretic extremities may lead to vasoconstriction by decreased shear flow. ${ }^{[1,3]}$ In clinical observations, the paretic extremities frequently revealed lower skin temperature than the non-paretic extremities in hemiplegic patients. ${ }^{[3]}$ Our previous investigations further demonstrated that paretic upper extremities (PUE) were associated with decreased hyperemic arterial inflow and endothelium-dependent cutaneous vasodilation in patients with stroke in the subacute stage. ${ }^{[4,5]}$ Moreover, dysfunction in cutaneous microcirculation tends to be more pronounced in the edematous than in the non-edematous extremities. $^{[4]}$

Edema refers to excessive accumulation of serous fluid in the extracellular spaces. It tends to develop in extremities that are in a dependent position or inactive owing to weakness, paralysis, or pain. ${ }^{[6]}$ Previous investigations found an incidence of hand edema, ranging from $16 \%$ to $82.5 \%$, following a stroke. ${ }^{[7]}$ Hand edema is likely to cause disfigured, disabling, reduced nutrition, contracture, and pain. ${ }^{[6,8]}$ Although preventing or alleviating edema of paretic extremities is critical in rehabilitating stroke patients, treatment with high-level evidence is currently unavailable (e.g., electrical stimulation). ${ }^{[9]}$ Faghri et al., investigated the immediate effect of neuromuscular electric stimulation (NMES) on hand edema after only one session of 30-min treatment in patients with stroke less than 6 months $(n=8)$, which demonstrated that hand edema was reduced compared to elevation. ${ }^{\left[{ }^{[1}\right.}$ Investigation regarding the effect of a repeated NMES intervention protocol on segmental fluid distribution of hemiplegic extremities is still lacking.

NMES is a common physical modality used for reconditioning the patients with stroke. This modality induces a repeated muscular contraction, possibly promoting the perfusion/oxygenation of the regional tissues. ${ }^{[10]}$ In addition to its effect on motor recovery facilitation and poststroke shoulder pain relief, ${ }^{[11,12]}$ literature regarding the effect of NMES on vascular property, segmental chemical composition, and fluid redistribution in paretic limbs is few. ${ }^{[8]}$ Our previous study showed that NMES enhances endothelial function in the paretic limbs in the patients with stroke. ${ }^{[5]}$ Additionally, NMES has been employed to enhance healing in pressure ulcers, venous ulcers, and diabetic ulcers. The primary effect of electrical stimulation on wound healing is due to an increase in skin perfusion by reducing sympathetic nervous system vasoconstriction of arteriolar smooth muscle, thus increasing skin blood flow. ${ }^{[10,13]}$ NMES is also used for the purpose of muscle strengthening, ${ }^{[14]}$ in which the protein synthesis is increased. Thus, regional chemical composition and hemodynamic properties may be altered by NMES. This passive muscle pumping might as well improve segmental fluid distribution in paralyzed musculature.

Therefore, repeated muscle contraction by NMES elicits regional metabolism and enhances local perfusion and oxygenation in the deconditioned limb, which mimics the effect of exercise. Accordingly, we hypothesize that NMES improves hemodynamic function, which is accompanied by modulated distribution of fluid to intra/extracellular milieu and chemical composition of segmental tissues in hemiplegic extremities of patients with stroke.

The aim of the present study was to evaluate the effects of 4-week NMES on arterial properties, fluid distribution, and body composition in PUE of patients who are being rehabilitated in the subacute stage after stroke. This work specifically used a rheographic pulse wave analysis to assess the hemodynamic property of artery, and simultaneously employed a multi-frequency bioelectrical impedance analysis to measure the distribution of fluid to intra/extracellular milieu and the chemical composition of segmental tissues.

\section{METHODS}

\section{Patients}

Stroke survivors admitted to the inpatient rehabilitation ward of a tertiary medical center (Chang Gung Memorial Hospital) within 6 weeks of their unilateral stroke were screened for inclusion. The inclusion criteria were as follows: (1) patients with severe upper extremity paresis (Brunnstrom stage I to II), (2) normal function in their nonparalytic upper extremities, (3) dependent ambulation, and (4) stable neurologic and medical status. All patients had a brain computed tomography scan, and the stroke was categorized as intracerebral hemorrhage or infarction. Paretic upper limbs of the participants were not considered edematous if they did not meet any of the following criteria: (1) dorsal swelling in the hand over the carpal bones; (2) fusiform edema of the metacarpophalangeal and proximal interphalangeal joints; (3) changes in temperature, color, or dryness; and (4) loss of dorsal skin lines and potential change of fingernails. ${ }^{[15]}$ Subjects were excluded if they had a history of significant cardiac arrhythmias, cardiac pacemaker implantation, post-stroke seizure, reflex sympathetic dystrophy, recurrent stroke, and any other condition that might have resulted in weakness of the paretic limbs. The institutional review board at Chang Gung Memorial Hospital approved this study. Written informed consent was obtained from all patients before the study.

\section{Intervention}

All patients underwent standard physical, occupational, and speech therapy as routine on a daily basis in the inpatient 
rehabilitation program. Additionally, all patients received 30 min of NMES on PUE daily and 5 days per week for 4 weeks. The patients received reciprocal electric stimulation for wrist extensors and flexors using two pairs of self-adhesive electrodes (NM III NMES; Rehabilicare, 1811 Old Hwy 8, New Brighton, MN 55112). ${ }^{[5]}$ On the extensor side, one electrode was placed proximally over the forearm $3 \mathrm{~cm}$ below the lateral epicondyle of humerus and the other was placed distally on the forearm $3 \mathrm{~cm}$ above the line across the radial and ulnar styloid process. On the flexor side, one electrode was placed proximally over the forearm $3 \mathrm{~cm}$ below the line across the medial and lateral epicondyle of the humerus and the other was placed distally on the forearm $3 \mathrm{~cm}$ above the proximal wrist crease [Figure 1a and b, supplementary]. The current intensity was set to produce reciprocal wrist and fingers' extension and flexion below the pain threshold, with a duty cycle of $10 \mathrm{sec}$ on and $5 \mathrm{sec}$ off. The stimulus pulse was a biphasic waveform with amplitude ranging from 10 to $40 \mathrm{~mA}$, a frequency at $35 \mathrm{~Hz}$, and a ramping up and down time of $2 \mathrm{sec}$ each. About 60 cycles of wrist extension and flexion occurred during each session. All treatments were carried out under the supervision of a single trained therapist using a standardized protocol..$^{[5]}$ The current intensity of electric stimulation and patient's compliance was recorded in a diary. Rheographic pulse wave and body composition analysis were performed in both PUE and non-paretic upper extremities (NPUE) on the same day prior to the first-time NMES treatment and on the next day after the last session. The measurements were carried out between 8 and 12 a.m. to avoid diurnal disturbance.

\section{Pulse wave analysis}

Baseline hemodynamic characteristics of NPUE and PUE were measured by impedance plethysmography (Rheo-

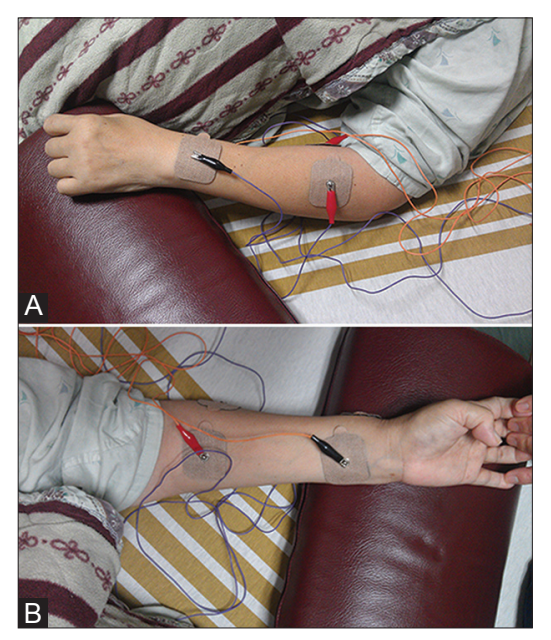

Figure 1: (A and B supplementary) The electrode placement of neuromuscular eletric stimulation on the extensor (A) and flexor (B) side of forearm in the paretic side. screen ${ }^{\circledR}$ compact; Medis, Ilmenau, Germany). ${ }^{[16]}$ Subjects were permitted $10 \mathrm{~min}$ of supine rest before testing. The measuring electrodes were placed on the flexor side of forearm on the same location as electric stimulation.

Basic impedance: Absolute impedance at the maximum of the pulse wave.

- Impedance quotient (IQ): Ratio of amplitude and basic impedance.

- Slope quotient (SQ): Ratio of maximal slope and basic impedance.

- Crest width (CW): Time delay between two curve points on either side of the curve maximum at a level of $95 \%$ of the amplitude.

- Crest time (CT): Time delay between the point of slope rise onset and the maximum of the curve.

- Propagation time (PT): Time delay between the R-peak in the ECG and the corresponding point of slope rise onset in the impedance signal.

- Alternating blood flow (ABF): The amount of blood pumping through the measuring segment during a heartbeat.

\section{Body composition analysis}

Maltron ${ }^{\circledR}$ BioScan 920-2S Analyser was employed. Baseline body composition characteristics of NPUE and PUE were measured by bioelectrical impedence analysis. ${ }^{[17,18]}$ Subjects were permitted $10 \mathrm{~min}$ of supine rest before testing. Low-level electrical currents, i.e. 5, 50, 100, $224 \mathrm{kHz}$, were passed through the body, and then impedance or opposition to flow of current was measured. This current at low frequencies $(5 \mathrm{kHz})$ passed through the extracellular fluids only; at higher frequencies, it penetrated cell membranes and passed through the intracellular fluid, as well as the extracellular fluids. Given that fat was anhydrous and a poor conductor of electrical current, the body impedance, measured at the constant frequency $50 \mathrm{kHz}$, primarily reflects the volume of the water and muscle compartments comprising the free fat mass (FFM) and the extracellular water volume. Accordingly, total body water (TBW), intracellular body water (ICW), and extracellular body water $(\mathrm{ECW})$ of extremities were determined in this study. Additionally, this method also analyzed chemical contents of protein, mineral, and glycogen, amount of muscle, fat, body cell mass (BCM), and ECM in non-paretic and paretic body sides.

\section{Statistical analysis}

Data were expressed as mean \pm SEM. The statistical software package StatView IV was used to analyze the data. Paired $t$-test was used to compare various parameters between PUE and NPUE before intervention. Repeated-measure analysis of variance (ANOVA) was 
employed to compare PUE and NPUE before and after 4 -week intervention. Differences were considered significant at $P<0.05$.

\section{RESULTS}

A total of 11 patients ( 5 men and 6 women), aged $62.6 \pm 12.5$ years, who had completed the NMES program were included in this study. Of them, seven had cerebral infarction and four had cerebral hemorrhage. The interval between stroke onset and rehabilitation was $15.6 \pm 6.6$ days. They received NMES treatment for $18.4 \pm 2.7$ times. No subject dropped out or had hand edema in either NPUE or PUE during intervention. All patients were admitted in the hospital throughout the intervention phase of this study. After 4-week follow-up, the Brunnstrom stage of PUE in the patients was all graded as II.

\section{Pulse wave analysis}

Before NMES treatment, the IQ, SQ, CT, CW, PT, and ABF of arterial pulse wave of PUE did not differ significantly from those of NPUE [Table 1]. After 4-week NMES treatment, NPUE had less decreased IQ, SQ, and ABF and less increased $\mathrm{CT}$ and $\mathrm{CW}$ of arterial pulse wave than those of PUE [Figure 2].

\section{Body water distribution}

Before NMES treatment, various body water contents, such as TBW, ECW, and ICW, did not differ significantly between NPUE and PUE [Table 1]. However, after NMES regimen, PUE had increased levels of TBW, ICW, and ECW in contrast to those in NPUE. Additionally, ECW/TBW in-

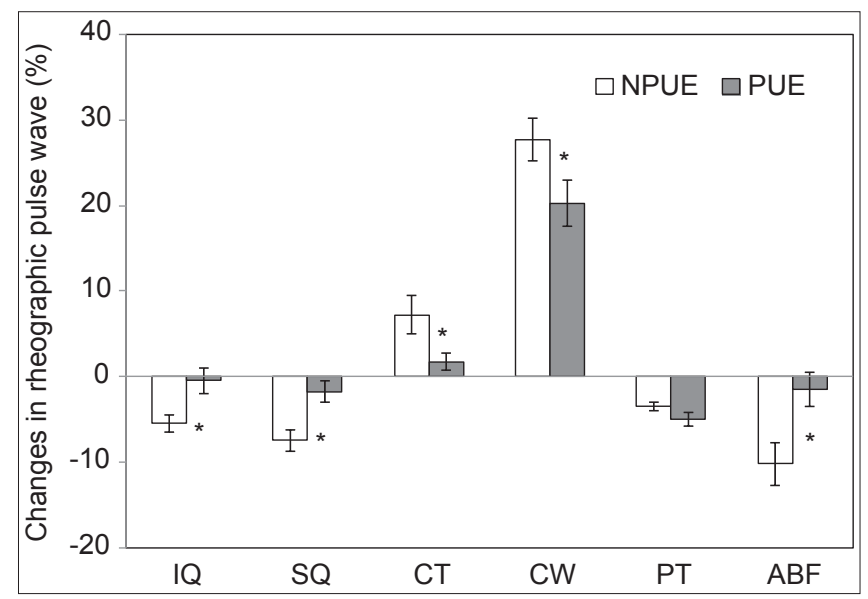

Figure 2: Changes in arterial pulse wave (\%) after neuromuscular electric stimulation, $* p<0.05$ (NPUE vs. PUE) (NPUE: Non-paretic upper extremity; PUE: Paretic upper extremity; IQ: Impedance quotient; SQ: Slope quotient; CT: Crest time; CW: Crest width; PT: Propagation time; ABF: Alternating blood flow; N: Non-paretic; P: Paretic). creased in both NPUE and PUE and did not differ between them after NMES treatment [Figure 3].

\section{Body composition}

Before NMES treatment, the body composition, including water, fat, protein, mineral, and glycogen of PUE did not

Table 1: Basic values of arterial pulse wave, body water contents, and body composition in non-paretic and paretic upper extremities of patients with hemiplegia before intervention

\begin{tabular}{lcc}
\hline & NPUE & PUE \\
\hline Arterial pulse wave & $0.73 \pm 0.21$ & \\
IQ (p.m.) & $11.2 \pm 2.1$ & $0.72 \pm 0.30$ \\
SQ (p.m./s) & $85.2 \pm 8.5$ & $9.3 \pm 2.5$ \\
CT (ms) & $40.1 \pm 5.1$ & $90.2 \pm 9.8$ \\
CW (ms) & $137 \pm 12$ & $41.9 \pm 4.2$ \\
PT (ms) & $42.5 \pm 5.1$ & $139 \pm 8$ \\
ABF (\%/min) & & $39.5 \pm 4.2$ \\
Body Water Contents & $48.5 \pm 4.2$ & \\
TBW (\%) & $17.2 \pm 3.1$ & $47.3 \pm 5.2$ \\
ECW (\%) & $31.2 \pm 4.2$ & $17.0 \pm 4.0$ \\
ICW (\%) & $35.2 \pm 3.2$ & $30.4 \pm 3.5$ \\
ECW/TBW (ratio) & & $35.0 \pm 4.3$ \\
Body composition & $32.1 \pm 3.2$ & \\
Fat (\%) & $9.2 \pm 1.9$ & $33.5 \pm 3.5$ \\
Protein (\%) & $4.1 \pm 0.9$ & $9.0 \pm 2.1$ \\
Glycogen (\%) & $5.8 \pm 1.4$ & $3.9 \pm 0.8$ \\
Mineral (\%) & $24.4 \pm 4.5$ & $5.6 \pm 1.2$ \\
BCM (\%) & $43.5 \pm 6.8$ & $21.3 \pm 5.1$ \\
ECM (\%) &
\end{tabular}

Abbreviations: PUE: paretic upper extremities; NPUE: Non-paretic upper extremities; BCM: Body cell mass; ECM: Extracellular mass; TBW: Total body water; ECW: Extracellular water; ICW: Intracellular water; IQ: Impedance quotient; SQ: Slope quotient; CT: Crest time; CW: Crest width; PT: Propagation time; ABF: Alternating blood flow; p.m. $=$ per mille

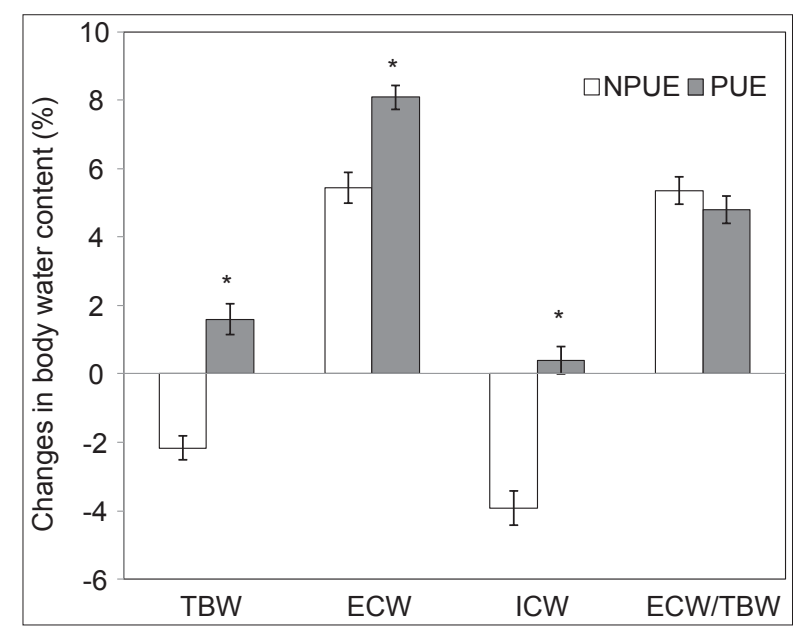

Figure 3: Changes in body water content (\%) after neuromuscular electric stimulation, $* p<0.05$ (NPUE vs. PUE) (TBW: Total body water; ECW: Extracellular water; ICW: Intracellular water). 
differ significantly from those of NPUE. Additionally, BCM and extracellular mass (ECM) showed no significant difference between both groups [Table 1]. However, PUE had higher level of protein, glycogen, mineral, and BCM, as well as lower level of fat than NPUE after NMES treatment [Figure 4].

\section{DISCUSSION}

To our knowledge, few studies addressed the effects of NMES on segmental fluid distribution and hemodynamic function in the extremities of patients with stroke. Compared to the sound side, $30 \mathrm{~min}$ of NMES, 5 days weekly for 4 weeks, had effects on the PUE as follows: (1) maintains the arterial inflow, IQ, SQ, CT, and CW in pulse wave analysis; (2) increases the percentage of intracellular water; (3) increases the BCM; and (4) decreases fat and increases protein, glycogen, and mineral composition percentage.

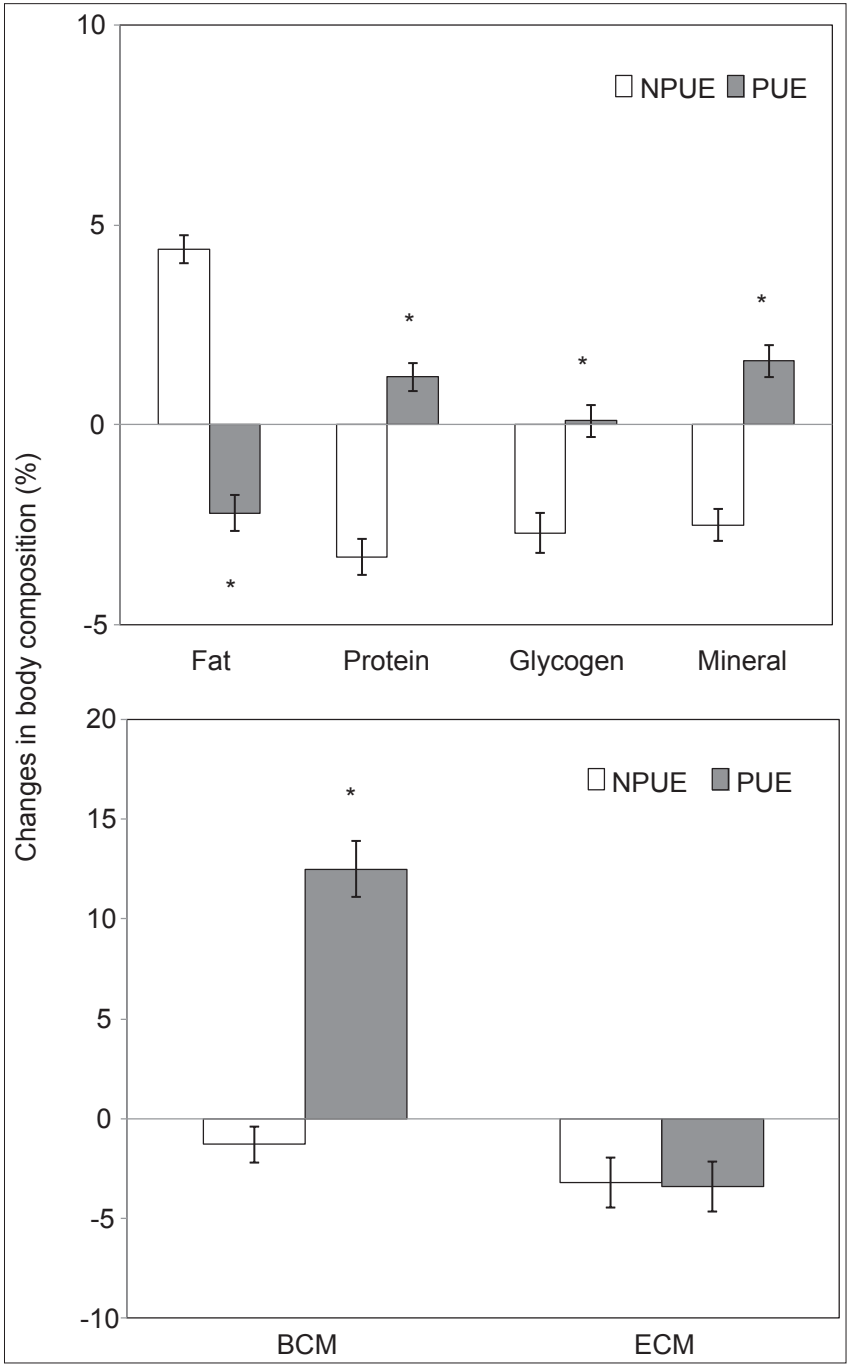

Figure 4: Changes in body composition (\%) after neuromuscular electric stimulation, $* p<0.05$ (NPUE vs. PUE) (BCM: Body cell mass; ECM: Extracellular mass).
These findings imply that NMES reverses the stroke-related decline of vascular property, segmental fluid distribution, and body composition in the subacute stage.

In NPUE, pulse wave analysis demonstrated increased $\mathrm{CW} / \mathrm{CT}$ and decreased arterial inflow/IQ/SQ during the 4-week experimental period, suggesting elevating vascular resistance. The results imply that although the NPUE can move freely, deconditioning of the extremities ${ }^{[1]}$ or stress-related systemic inflammation in the subacute stage of stroke ${ }^{[19]}$ might impair vascular function. Physiological hemodynamic shear stress that is caused by increased blood flow, even for a few minutes, increases the secretion of nitric oxide and prostacyclin, both of which enhance vasodilatory function. ${ }^{[20]}$ Cardiovascular deconditioning results in decreased blood flow, causing impaired endothelial function and, thereby, elevating basal arterial tone. ${ }^{[1]}$

On the other hand, the current study showed that NMES maintained the arterial property in the PUE. Similar to our previous study, endothelial function and cutaneous microcirculation in the paretic limb can as well be maintained by NMES to prevent its decline as the sound side. ${ }^{[4,5]} \mathrm{In}$ the paretic extremities, NMES seems to be an appropriate treatment in the flaccid stage of stroke. Besides, it can be conveniently performed in the clinical setting. In rehabilitation program for stroke patients, treatment mostly focuses on paretic limbs. However, physical deconditioning of the non-paretic limbs is common because their daily activities are markedly reduced. The results herein imply that rehabilitation programs should incorporate resistance training for non-paretic limbs.

BCM is the total mass of all the cellular elements in the body which constitute all the metabolically active tissues of the body. Depletion of BCM is characteristic of wasting. ${ }^{[21,22]}$ Similar to this experimental finding, Dr. Lone Jørgensen found that lean muscle mass is rapidly lost after acute stroke. Moreover, a significant 5\% loss of lean mass was found at 2 months on the non-paretic leg after acute stroke. ${ }^{[23]}$ The result showed that after 4-week NMES treatment in the PUE, BCM as well as ICW, which is a component of BCM, increased. ${ }^{[24]}$ Furthermore, the current study demonstrated that NMES intervention, a repetitive passive muscle contraction, led to favorable regional nutritional status by replacing fat tissue with protein and mineral and, thereby, retarded muscle wasting and demineralization as shown in the NPUE.

Dependent edema of the hand, sometimes extending up the arm, occurs in stroke patients. Edema is prone to occur if the upper extremity is completely paralyzed ${ }^{[8]}$ After 4-week follow-up, TBW and ICW were decreased while ECW was increased in the NPUE. On the contrary, 4-week NMES regimen elevated TBW, ECW, and ICW, probably due to increased regional metabolism by passive muscle contrac- 
tion. Nonetheless, ECW/TBW showed no difference in both groups, suggesting the osmotic gradient between the cell membrane is unaltered. Although the present NMES protocol failed to reduce ECW or ECW/TBW in PUE compared to NPUE, it elevated ICW and BCM and maintained the segmental tissue in a favorable fluid and nutritional status.

Small sample size weakened its statistical power. Larger sample size is needed in further studies and NMES may be as well applied in lower extremities. In addition, minimal effective protocol of NMES required to improve the vascular properties and body composition may be determined. Also, the effect of NMES may be multi-factorial and require further study to clarify its mechanisms. The experimental findings might only be applicable to patients with subacute stroke less than 6 weeks and with flaccid upper extremities (Brunnstrom stage I to II).

\section{Conclusion}

NMES delays the stroke-related decline of peripheral vascular function and extremity fluid redistribution in the PUE of patients with hemiplegia. It may be considered for stroke patients with hemiplegia when there are no other medical contraindications. In addition, resistance training for the non-paretic extremities may be included in stroke rehabilitation programs to attenuate the decline of hemodynamic function.

\section{REFERENCES}

1. Coupe M, Fortrat JO, Larina I, Gauquelin-Koch G, Gharib C, Custaud MA. Cardiovascular deconditioning: From autonomic nervous system to microvascular dysfunctions. Respir Physiol Neurobiol 2009;169 Suppl 1:S10-2.

2. Rimmer JH, Schiller W, Chen MD. Effects of disability-associated low energy expenditure deconditioning syndrome. Exerc Sport Sci Rev 2012;40:22-9.

3. Adams WC, Imms FJ. Resting blood flow in the paretic and nonparetic lower legs of hemiplegic persons: Relation to local skin temperature. Arch Phys Med Rehabil 1983;64:423-8.

4. Wang JS, Yang CF, Liaw MY, Wong MK. Suppressed cutaneous endothelial vascular control and hemodynamic changes in paretic extremities with edema in the extremities of patients with hemiplegia. Arch Phys Med Rehabil 2002;83:1017-23.

5. Wang JS, Chen SY, Lan C, Wong MK, Lai JS. Neuromuscular electric stimulation enhances endothelial vascular control and hemodynamic function in paretic upper extremities of patients with stroke. Arch Phys Med Rehabil 2004;85:1112-6.

6. Sorenson MK. The edematous hand. Phys Ther 1989;69:1059-64.

7. Gebruers N, Truijen S, Engelborghs S, De Deyn PP. Is activity loss predictive for development of upper limb oedema after stroke? J Rehabil Med 2011;43:398-403.

8. Faghri PD. The effects of neuromuscular stimulation-induced muscle contraction versus elevation on hand edema in CVA patients. J Hand Ther 1997;10:29-34.
9. Gustafsson L, Yates K. Are we applying interventions with research evidence when targeting secondary complications of the stroke-affected upper limb. Aust Occup Ther J 2008;56:428-35.

10. Gilcreast DM, Stotts NA, Froelicher ES, Baker LL, Moss KM. Effect of electrical stimulation on foot skin perfusion in persons with or at risk for diabetic foot ulcers. Wound Repair Regen 1998;6:434-41.

11. Yavuzer G, Geler-Kulcu D, Sonel-Tur B, Kutlay S, Ergin S, Stam HJ. Neuromuscular electric stimulation effect on lower-extremity motor recovery and gait kinematics of patients with stroke: A randomized controlled trial. Arch Phys Med Rehabil 2006;87:536-40.

12. Yu DT, Chae J, Walker ME, Kirsteins A, Elovic EP, Flanagan SR, et al. Intramuscular neuromuscular electric stimulation for poststroke shoulder pain: A multicenter randomized clinical trial. Arch Phys Med Rehabil 2004;85:695-704.

13. Rutherford RB, Shannon FL. Lumbar sympathectomy: Indications and technique. Vascular surgery. In: Rutherford RB, editor, $4^{\text {th }}$ ed. Philadelphia: Saunders; 1995.

14. Sillen MJ, Speksnijder CM, Eterman RM, Janssen PP, Wagers SS, Wouters EF, et al. Effects of neuromuscular electrical stimulation of muscles of ambulation in patients with CHF or COPD: A systematic review of the English-language literature. Chest 2009;136:44-61.

15. Geurts AC, Visschers BA, van Limbeek J, Ribbers GM. Systematic review of aetiology and treatment of post-stroke hand oedema and shoulder-hand syndrome. Scand J Rehabil Med 2000;32:4-10.

16. Huang SC, Wong MK, Wang JS. Systemic hypoxia affects cardiac autonomic activity and vascular hemodynamic control modulated by physical stimulation. Eur J Appl Physiol 2009;106:31-40.

17. Liu MH, Wang CH, Huang YY, Tung TH, Lee CM, Yang NI, et al. Edema index established by a segmental multifrequency bioelectrical impedance analysis provides prognostic value in acute heart failure. J Cardiovasc Med 2012;13:299-306.

18. Lewy VD, Danadian K, Arslanian S. Determination of body composition in African-American children: Validation of bioelectrical impedence with dual energy X-ray absorptiometry. J Pediatr Endocrinol Metabol 1999;12:443-8.

19. Beer C, Blacker D, Hankey GJ, Puddey IB. Association of clinical and aetiologic subtype of acute ischaemic stroke with inflammation, oxidative stress and vascular function: A cross-sectional observational study. Med Sci Mon Int Med J Exp Clin Res 2011;17:CR467-73.

20. Wray DW, Witman MA, Ives SJ, McDaniel J, Fjeldstad AS, Trinity JD, et al. Progressive handgrip exercise: Evidence of nitric oxide-dependent vasodilation and blood flow regulation in humans. Am J Physiol Heart Circ Physiol 2011;300:H1101-7.

21. Chen YM, Chen HH, Hsieh CW, Hsieh TY, Lan JL, Chen DY. A close association of body cell mass loss with disease activity and disability in Chinese patients with rheumatoid arthritis. Clinics (Sao Paulo, Brazil) 2011;66:1217-22.

22. Roubenoff R. The pathophysiology of wasting in the elderly. J Nutr 1999;129:256-9S.

23. Jorgensen L, Jacobsen BK. Changes in muscle mass, fat mass, and bone mineral content in the legs after stroke: A 1 year prospective study. Bone 2001;28:655-9.

24. Ha L, Hauge T, Iversen PO. Body composition in older acute stroke patients after treatment with individualized, nutritional supplementation while in hospital. BMC Geriatr 2010;10:75. 